\title{
$\angle S$ Research Suare \\ Effect of Neutrophil CD64 Index in Elderly Patient with Community-acquired Pneumonia
}

Jie Cao ( $\sim$ TJZYYcaojie2021@163.com )

Tianjin medical university

YAN LI

Tianjin Medical University

Suhang-Wang

Tianjin Medical University

Jing-Zhang

Tianjin Medical University

\section{Research}

Keywords: community-acquired pneumonia, neutrophil CD64 index, C-reactive protein, procalcitonin

Posted Date: August 23rd, 2021

DOI: https://doi.org/10.21203/rs.3.rs-801021/v1

License: (9) This work is licensed under a Creative Commons Attribution 4.0 International License. Read Full License 


\section{Abstract}

Background: Elderly patient with community-acquired pneumonia is the leading infectious cause of death. During the clinical diagnosis and treatment, some elderly patients do not have typical clinical symptoms . Therefore establishment of safe and effective diagnosis, prognostic assessment systems is important for clinicians.

Objective: To evaluate the diagnostic and prognostic value of the neutrophil CD64(nCD64) in elderly patients with community-acquired pneumonia.

Methods: One hundred and twenty-eight elderly patients( $\geq 65$ year) diagnosed with community-acquired pneumonia from December 2018 to December 2020. All patients were further subdivided into two groups: Non severe community-acquired pneumonia(N-SCAP) group and severe community-acquired pneumonia (SCAP)group. nCD64 index, procalcitonin (PCT) level, C-reactive protein (CRP) level, White blood cell (WBC) counts and Neutrophil (NEUT) absolute counts were obtained and CURB-65 scores were calculated for each patient.

Results: The nCD64, CRP ,PCT, WBC,NEUT levels,CURB-65 score were higher in severe communityacquired pneumonia group patients. The nCD64, CRP, PCT levels ,CURB-65 score were higher in nonsurvivors. The receiver operating characteristic (ROC) curve of $\mathrm{nCD} 64$ was higher than those of CRP, PCT, WBC, NEUT levels for diagnosing infection. The AUC of nCD64 for predicting 28-day mortality in community-acquired pneumonia was significantly higher than those of CRP ,PCT, WBC and NEUT. The AUC of nCD64 combined with CURB-65 score was significantly higher than that of CRP, PCT, WBC and NEUT parameter combined with CURB-65 score for predicting 28-day mortality.

Conclusions: The neutrophil CD64 index is a valuable biomarker for diagnosis of infection and prognostic evaluation in elderly patients ( $\geq 65$ year) with community-acquired pneumonia.

\section{Introduction}

Community-acquired pneumonia (CAP) is an infectious inflammation of the lung parenchyma that occurs outside the hospital, including pathogen infection with a specific incubation period and onset within the average incubation period after admission. It is defined as the presence of a lung infiltration shadow on chest radiography and any symptoms such as cough, sputum, fever, dyspnea, and chest pain ${ }^{[1]}$. Severe pneumonia can occur in both community-acquired pneumonia (CAP) and hospital-acquired pneumonia (HAP). Severe pneumonia is identified in pneumonia patients with continuous hypoxemia or acute respiratory failure requiring ventilation support, circulatory failure such as hypotensive shock, or other organ dysfunction. In this study, severe community-acquired pneumonia (SCAP)was studied. CAP is a major public health problem with high morbidity, mortality and short and long-term sequelae. In adults, the incidence of CAP and related hospitalization and mortality increase steadily with age, with a dramatic rise after the age of 65. In developed countries, almost one half of the total hospitalizations for CAP occur in patients over 65 years old ${ }^{[2]}$. The severity of CAP also increases with age, primarily due to age-related 
immune dysfunction, and greater likelihood of underlying comorbid factors in elderly patients ${ }^{[3]}$. In recent years, there has been a steady increase in the hospitalization rates including intensive care units (ICU) due to SCAP, especially in the elder population ${ }^{[4]}$. The immuneosenescence comorbidities and frailty of these patients increases their susceptibility to infectious diseases. It is the leading infectious cause of death and the fourth overall cause of mortality in the elderly ${ }^{[5]}$. During the clinical diagnosis and treatment, some CAP patients, especially elderly patients, do not have cough, sputum, fever. Therefore, we usually perform blood tests for biomarkers to differentiate CAP from other non-infectious respiratory diseases. The blood culture remains the gold standard for infection diagnosis, even though its result is usually delayed for more than 48 hours. Additionally, there are false-positive results due to the impossibility of excluding contamination, besides its false-negative results which are frequently encountered in the elder population due to small unsatisfactory blood sample volume encountered in many circumstances. Despite the routine use of infection markers such as white blood count (WBC), Creactive protein (CRP), and procalcitonin (PCT), there are many confounding factors, false positives, and false negatives which make them less ideal. As a result, in the past few years, attention has been directed to other sepsis biomarkers including leukocyte cell surface antigens ${ }^{[6]}$.

Neutrophil CD64 (nCD64) is leukocyte cell surface antigens. Neutrophil CD64 known as Fc receptor 1 (FcR1), is a high-affinity receptor present on neutrophils for Fc part of immunoglobulin-G (IgG) heavy chain ${ }^{[7-8]}$.Its expression gets strongly upregulated in response to proinflammatory cytokines of infection within 4-6 hours. Several studies have indicated that neutrophil CD64 (nCD64) index is a highly sensitive and specific marker for the diagnosis of sepsis of bacterial origin and differentiating sepsis from nonseptic conditions. Neutrophil CD64 index is an emerging novel biomarker with prognostic implications in critically ill patients. Extensive literature search has revealed that $\mathrm{nCD} 64$ index is a valuable marker for the early diagnosis of patients with sepsis both in emergency department and ICU. Some prospective studies and meta-analysis have documented that nCD64 index has a very good sensitivity and specificity for the diagnosis of sepsis $(80-90 \%)^{[9]}$. Its diagnostic accuracy is definitely better than conventional biomarkers for sepsis including the most widely used biomarker PCT and CRP, with better AUROC in most of the studies. Apart from a useful established diagnostic marker, it has its own prognostic implications. Neutrophil CD64 index has been found to be a predictor of outcome during ICU stay in the form of survival or mortality and an early predictor of impending clinical deterioration. However, only a few studies have evaluated the diagnostic and prognostic utility of nCD64 in elderly patients with communityacquired pneumonia.

In this study, we used a single-center, retrospective clinical study design to explore the diagnostic and prognostic significance of nCD64 in elderly patients with community-acquired pneumonia. The aim is to assess the effect of the nCD64 in elderly patients with community-acquired pneumonia.

\section{Methods}

\section{Subject data collection}


A single-center retrospective study was conducted on 128 elderly patients with community-acquired pneumonia in Tianjin Hospital of Integrated Chinese and Western Medicine from December 2018 to December 2020.All selected patients were diagnosed with community-acquired pneumonia according to the diagnostic criteria in "Diagnosis and Treatment of Community-Acquired Pneumonia in China" [10]:1. The onset of the community; 2. Pneumonia-related clinical manifestations: (1) new cough or sputum symptoms, or exacerbation of symptoms or purulent sputum on the basis of existing cough or sputum; (2) fever; (3) pulmonary consolidation signs and/or wet rales;(4) Peripheral white blood cells $\geq 10 \otimes 10^{9} / \mathrm{L}$ or $\leq 4 \llbracket 10^{9} / \mathrm{L}$, with or without nucleus left shift; 3 . Chest imaging examination revealed new patchy infiltrating shadows, leaf/segment consolidation shadows, ground glass shadows, or interstitial changes with or without pleural effusion. Have $1+3$ and any item of 2. Diagnostic criteria for severe pneumonia are met: Main diagnoses: (1) Mechanical ventilation for endotracheal intubation; (2) Septic shock still requires vasoactive drug therapy after active fluid resuscitation. Secondary criteria: (1) Respiratory rate $\geq 30$ times/min; (2) Oxygenation index $\leq 250 \mathrm{mmHg}$;(3) Multiple lobar infiltrates; (4) Disturbance of consciousness;(5) Blood urea nitrogen $\geq 7.14 \mathrm{mmol} / \mathrm{L}$; (6) Systolic blood pressure $\leq 90 \mathrm{mmHg}$ requires after aggressive fluid resuscitation. Severe pneumonia can be diagnosed if one of the following primary diagnoses is met or $\geq 3$ secondary criteria are met.

The following patients were excluded: (1) patients <65 years old; (2) pulmonary tuberculosis ;(3) pulmonary tumor;(4) atelectasis;(5) pulmonary embolism;(6) pulmonary eosinophilic infiltration;(7) pulmonary vasculitis;(8) Patients with infection of other parts, trauma, surgery, long-term dialysis and serious diseases of other organs.

On admission to the hospital the following items were recorded for each selected patient: age, sex, medical history, comorbidities, All patients were followed up for up to 28 days, with the primary endpoint being 28-day mortality.

\section{Analysis of laboratory biomarkers}

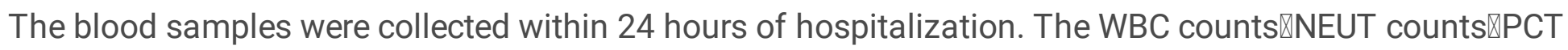
level and CRP level information from enrolled patients were recorded on admission. WBCロNEUT absolute were measured via an automated hematology analyzer (Sysmex XS-500i, Sysmex Corporation Kobe, Japan). The concentrations of serum procalcitonin (PCT) were measured using a BioMerieux Mini VIDAS immunoassay analyzer (Block Scientific, Bohemia, NY, USA), and the limit of detection (LOD) was $0.05 \mathrm{ng} / \mathrm{ml}$ Serum C-reactive protein (CRP) concentrations were analyzed utilizing turbidimetric immunoassay (BNII, Siemens Healthcare Diagnostic, Germany). Neutrophil CD64 (nCD64) was measured using flow cytometry using a commercial kit (Quanti BRITE PE, Becton Dickinson). Briefly, phosphatebuffered saline-diluted whole blood $(100 \mu \mathrm{L})$ was incubated for 20 minutes at room temperature with a combination of anti-CD14-FITC and anti-CD64-PE. After lysis, blood samples were washed and fixed with BD Lyse/Wash Assistant. Neutrophils were identified by electronic gating based forward and side scatter. Interassay standardization and CD64 quantization were performed using Quanti BRITEPE calibration 
beads with known numbers of PE molecules. Data analysis was performed by using light scatter gating to define the neutrophil population, and the nCD64 was quantified as mean equivalent soluble fluorescence units using BD Diva software.

The study protocol was approved by the hospital's ethics committee. Written informed consent was obtained from either the patients or their legal surrogates.

\section{Statistical analysis}

Statistical analyses were performed with SPSS version 24.0 (IBM Corp, Armonk, NY, USA). Descriptive results of continuous variables were expressed as mean and standard deviation or median and interquartile range depending of the normality of their distribution. Variables were tested for their association with the diagnosis using Pearson's $\chi 2$ test for categorical data. Receiver operating characteristic (ROC) curves were used to compare the predictive prognostic efficacy of the nCD64, CRPQ PCT ,WBC, NEUT , CURB-65 score and to calculate their area under the curves (AUC) was calculated using MedCalc 15.0 Software (Acacialaan, Ostend, Belgium) for diagnostic power. A z-test was used to compare AUCs between different curves. Binary logistic regression was performed to analyze the risk factors of 28-day mortality. Statistical significance was set at $P<0.05$ (two-sided).

\section{Results}

\section{Clincal characteristics of patients}

From December 2018 to December 2020,128 community-acquired pneumonia patients were further subdivided into two groups: Non severe community-acquired pneumonia(N-SCAP) group $(n=96)$ and severe community-acquired pneumonia (SCAP) group ( $n=32)$. N-SCAP group: The mean age of patients was $74.78( \pm 9.49)$ years, $55 \%$ were males, $45 \%$ were Females, $59.4 \%$ smoked, $12.5 \%$ Heavly alcohol consumption. Comorbidities: Coronary heart disease (66.7\%) ,Hypertension (58.3\%),Diabetes mellius (62.5\%),Hyperlipemia (63.5\%). SCAP group: The mean age of patients was $75.69( \pm 9.73)$ years, $59.4 \%$ were males, $40.6 \%$ were Females ,50\% smoked, $3.1 \%$ Heavly alcohol consumption. Comorbidities: Coronary heart disease (68.8\%) ,Hypertension (65.5\%),Diabetes mellius (68.8\%),Hyperlipemia (71.9\%). The characteristics of patients in N-SCAP group and SCAP group had no significant difference $(P>0.05)$ (Table 1).

\section{Table 1}

Baseline characteristics of patients based on N-SCAP and SCAP 


\begin{tabular}{|c|c|c|c|}
\hline Characteristics & $\mathrm{N}-\mathrm{SCAP}(\mathrm{N}=96)$ & $\operatorname{SCAP}(\mathrm{N}=32)$ & $P$ value N-SCAP versus SCAP \\
\hline Age, years & $74.78 \pm 9.49$ & $75.69 \pm 9.73$ & 0.605 \\
\hline Male(\%) & $55 \llbracket 57.3 \% \rrbracket$ & $19 ه 59.4 \% \square$ & 0.836 \\
\hline Smoking(\%) & $57 \rrbracket 59.4 \% \bigotimes$ & $16 \rrbracket 50 \% \rrbracket$ & 0.354 \\
\hline Heavly alcohol consumption(\%) & $12 \llbracket 12.5 \% \bigotimes$ & $1 \rrbracket 3.1 \% \bigotimes$ & 0.128 \\
\hline \multicolumn{4}{|l|}{ Comorbidities(\%) } \\
\hline Coronary heart disease & $64 \llbracket 66.7 \% \rrbracket$ & $22 \rrbracket 68.8 \% \rrbracket$ & 0.828 \\
\hline Hypertension & $56 \llbracket 58.3 \% \rrbracket$ & $21 \rrbracket 65.5 \% \bigotimes$ & 0.466 \\
\hline Diabetes mellius & $60 \rrbracket 62.5 \% \rrbracket$ & $22 \rrbracket 68.8 \% \bigotimes$ & 0.523 \\
\hline Hyperlipemia & 61囚63.5\%区 & $23 \bowtie 71.9 \% \rrbracket$ & 0.390 \\
\hline
\end{tabular}

\section{Comparison of nCD64 ,CRP ,PCT, WBC,NEUTXCURB-65 score in SCAP and N-SCAP groups}

nCD 64, CRP ,PCT, WBC,NEUT level as well as CURB-65 score were higher in SCAP patients compared with N-SCAP patients (Figure 1). Significant differences between the SCAP and N-SCAP groups (Table 2).

Table 2

nCD64, CRP, PCT, WBC, NEUT levels and bacterial infection in N-SCAP and SCAP 


\begin{tabular}{|llll|}
\hline Characteristics & N-SCAP $(\mathrm{n}=96)$ & $\mathrm{SCAP}(\mathrm{n}=32)$ & P value N-SCAP versus SCAP \\
\hline CURB-65 & $2.22 \pm 1.32$ & $4.06 \pm 1.13$ & 0.000 \\
\hline nCD $64 \%$ & $6.14 \pm 4.46$ & $11.91 \pm 6.85$ & 0.000 \\
\hline PCT $(\mathrm{ng} / \mathrm{ml})$ & $2.27 \pm 2.25$ & $5.62 \pm 3.37$ & 0.000 \\
\hline CRP $(\mathrm{mg} / \mathrm{ml})$ & $45.47 \pm 41.36$ & $76.86 \pm 43.09$ & 0.000 \\
\hline WBC $(\times 109 / \mathrm{L})$ & $9.95 \pm 3.31$ & $12.62 \pm 5.39$ & 0.012 \\
\hline NEUT $(\times 109 / \mathrm{L})$ & $7.12 \pm 3.55$ & $9.17 \pm 4.06$ & 0.007 \\
\hline Microbiological etiology & & & \\
\hline Streptococcus pneumoniae & 8 & 7 & \\
\hline Haemophilus influenzae & 4 & 0 & \\
\hline Pseudomonas aeruginosa & 3 & 6 & \\
\hline Klebsiella Pneumoniae & 2 & 5 & \\
\hline Other microorganism & 1 & 2 & \\
\hline
\end{tabular}

\section{Evaluation of nCD64, CRP, PCT, WBC, NEUT levels and in diagnosing bacterial infection}

The statistical values of ROC curves for nCD64, CRP, PCT, WBC, NEUT in differentiating a positive microbial culture from CAP are shown in (Table 5). nCD64 produced the highest AUC (0.787), followed by CRP (0.681), PCT (0.629). There were significant differences between nCD64 and CRP or PCT $(P<0.05)$, but there were no significant differences between WBC $(P=0.325)$ and NEUT $(P=0.077)$.

\section{Comparison between patient survivors and non-survivors at the 28-day follow-up}

Baseline characteristics are shown in (Table 3). Among the patients enrolled, 109 survived and 19 died at the 28-day follow-up. The mortality rate was $14.8 \%$ (19/128). The nCD64,CRP,PCT levels,CURB-65 score were higher in non-survivors. There were significant differences in nCD64, but no significant difference in WBC, NEUT count between survivors and non-survivors.

\section{Prognostic value of nCD64, CRP, PCT, WBC , NEUT levels and CURB-65 score}


The ROC curves of nCD64, PCT, CRP, WBC, NEUT, CURB-65 score for predicting death are shown in (Figure 2). nCD64 had the highest AUC (0.907), followed by CRP (0.710), PCT (0.678), WBC (0.592) and NEUT (0.573) (Table 4, Figure 2). There were significant differences between $\mathrm{nCD} 64$ and CRP or PCT $(P<0.001)$, but there were no significant differences between WBC $(P=0.276)$ and NEUT $(P=0.412)$. The combination of nCD64 +CURB-65 score achieved an AUC of (0.905), followed by the combination of CRP+ CURB-65 score achieved an AUC of (0.712), PCT +CURB-65 score achieved an AUC of (0.704), WBC +CURB-65 score achieved an AUC of (0.632), NEUT +CURB-65 score achieved an AUC of (0.636). There were significant differences between nCD64+CURB-65score,CRP+CURB-65score,PCT+CURB-65 score $(P<0.001)$,CURB-65score $(P=0.005)$. There was no significant difference between WBC+CURB-65 score $(P=0.101)$,NEUT+CURB-65 score $(P=0.636)$. (Table 5 ,Figure 3$)$. Binary logistic regression analysis revealed that nCD 64 were independent risk factors of 28-day mortality in patients with sepsis (Table 6).

Table 3

Baseline characteristics of patients based on outcome

\begin{tabular}{|llll|}
\hline Variables & Survival $(\mathrm{n}=109)$ & Death $(\mathrm{n}=19)$ & P value \\
\hline Age, years & $75.08 \pm 9.85$ & $74.58 \pm 7.52$ & 0.800 \\
\hline Sex $(\%)$ & & & 0.994 \\
\hline Male & $63(49.2 \%)$ & $11(8.6 \%)$ & \\
\hline Female & $46(35.9 \%)$ & $8(6.3 \%)$ & \\
\hline Smoking & $58(45.3 \%)$ & $15(11.7 \%)$ & 0.037 \\
\hline Heavly alcohol consumption & $12(9.4 \%)$ & $1(0.8 \%)$ & 0.444 \\
\hline Comorbidities(\%) & & & \\
\hline Coronary heart disease & $73(57 \%)$ & $13(10.2 \%)$ & 0.901 \\
\hline Hypertension & $65(50.8 \%)$ & $12(9.4 \%)$ & 0.772 \\
\hline Diabetes mellius & $69(53.9 \%)$ & $13(10.2 \%)$ & 0.668 \\
\hline Hyperlipemia & $71(55.5 \%)$ & $13(10.2 \%)$ & 0.781 \\
\hline CURB-65 & $2.53 \pm 1.48$ & $3.53 \pm 1.43$ & 0.007 \\
\hline CD64(MFI) & $6.23 \pm 4.78$ & $14.89 \pm 4.91$ & 0.000 \\
\hline PCT(ng/mL) & $2.82 \pm 2.73$ & $4.80 \pm 3.59$ & 0.006 \\
\hline CRP(mg/L) & $50.04 \pm 44.25$ & $72.14 \pm 36.78$ & 0.042 \\
\hline WBC $\left(\times 10^{9} / \mathrm{L}\right)$ & $10.43 \pm 3.91$ & $11.68 \pm 4.91$ & 0.218 \\
\hline NENT $\left(\times 10^{9} / \mathrm{L}\right)$ & $7.43 \pm 3.56$ & $8.77 \pm 4.81$ & 0.260 \\
\hline
\end{tabular}


Table 4

Analysis of ROC curves in predicting 28-day mortality in patients with CAP

\begin{tabular}{|llllllll|}
\hline Variables & AUC $(95 \% \mathrm{Cl})$ & Pvalue & $\begin{array}{l}\text { Cut-off } \\
\text { value }\end{array}$ & Sensitivity & specificity & PPV & NPV \\
\hline CD64 & $\begin{array}{l}0.907(0.843- \\
0.951)\end{array}$ & $<0.0001$ & $>10.43$ & 0.100 & 0.798 & 0.463 & 0.100 \\
\hline CRP & $\begin{array}{l}0.710(0.623- \\
0.758)\end{array}$ & 0.0004 & $>56.77$ & 0.684 & 0.743 & 0.316 & 0.931 \\
\hline PCT & $\begin{array}{l}0.678(0.590- \\
0.758)\end{array}$ & 0.0070 & $>2.65$ & 0.790 & 0.633 & 0.272 & 0.945 \\
WBC & $\begin{array}{l}0.592(0.501- \\
0.678)\end{array}$ & 0.276 & $>12.87$ & 0.474 & 0.771 & 0.264 & 0.894 \\
\hline AUC: area under the curve; PPV: positive predictive value; NPV: negative predictive value; & \\
\hline
\end{tabular}

Table 5

Analysis of ROC curves in diagnosing positive infection culture in patients with CAP 


\begin{tabular}{|c|c|c|c|c|c|c|c|}
\hline Variables & AUC (95\%Cl) & $\mathrm{P}$ value & $\begin{array}{l}\text { Cut-off } \\
\text { value }\end{array}$ & Sensitivity & specificity & PPV & NPV \\
\hline CD64 & $\begin{array}{l}0.787(0.381- \\
0.703)\end{array}$ & $<0.001$ & $>8.95$ & 0.743 & 0.820 & 0.645 & 0.879 \\
\hline CRP & $\begin{array}{l}0.681(0.108- \\
0.408)\end{array}$ & $<0.001$ & $>28.67$ & 0.871 & 0.438 & 0.405 & 0.886 \\
\hline РCT & $\begin{array}{l}0.629(0.149- \\
0.477)\end{array}$ & 0.020 & $>3.34$ & 0.615 & 0.708 & 0.480 & 0.807 \\
\hline WBC & $\begin{array}{l}0.557(0.089- \\
0.280)\end{array}$ & 0.325 & $>10.33$ & 0.590 & 0.584 & 0.384 & 0.764 \\
\hline NEUT & $\begin{array}{l}0.605(0.515- \\
0.691)\end{array}$ & 0.077 & $>9.34$ & 0.513 & 0.753 & 0.477 & 0.779 \\
\hline $\begin{array}{l}\text { CD64+CURB- } \\
65\end{array}$ & $\begin{array}{l}0.905(0.840- \\
0.949)\end{array}$ & $<0.0001$ & $>12.23$ & 0.1 & 0.762 & 0.421 & 0.100 \\
\hline $\begin{array}{l}\text { CRP+CURB- } \\
65\end{array}$ & $\begin{array}{l}0.712(0.840- \\
0.949)\end{array}$ & 0.0004 & $>61.33$ & 0.684 & 0.752 & 0.324 & 0.932 \\
\hline $\begin{array}{l}\text { PCT+CURB- } \\
65\end{array}$ & $\begin{array}{l}0.704(0.173- \\
0.582)\end{array}$ & 0.0012 & $>6.36$ & 0.737 & 0.679 & 0.341 & 0.943 \\
\hline $\begin{array}{l}\text { WBC+CURB- } \\
65\end{array}$ & $\begin{array}{l}0.632(0.164- \\
0.517)\end{array}$ & 0.1011 & $>15.26$ & 0.579 & 0.762 & 0.297 & 0.912 \\
\hline $\begin{array}{l}\text { NEUT+CURB- } \\
65\end{array}$ & $\begin{array}{l}0.636(0.174- \\
0.526)\end{array}$ & 0.088 & $>12.34$ & 0.579 & 0.762 & 0.297 & 0.912 \\
\hline CURB-65 & $\begin{array}{l}0.680(0.591- \\
0.759)\end{array}$ & 0.005 & $>2$ & 0.684 & 0.633 & 0.225 & 0.920 \\
\hline
\end{tabular}

Table 6

Independent predictive variables analysis by multivariate logistic regression

\begin{tabular}{|llllll|}
\hline variable & coefficient & wald & $P$ value & Adjusted OR & $95 \% \mathrm{Cl}$ \\
\hline nCD64 & 0.309 & 19.90 & $<0.0001$ & 1.362 & $1.1891-1.560$ \\
\hline constant & -4.883 & 31.45 & $<0.0001$ & & \\
\hline
\end{tabular}

\section{Discussion}

Community-acquired pneumonia (CAP) is the most prevalent infectious disease in the elderly patient and is associated with high rates of mortality, morbidity and high costs worldwide ${ }^{[11-12]}$. Therefore, 
establishment of safe and effective diagnosis prognostic assessment systems of CAP is important for clinicians. Commonly used biomarkers for early diagnosis of bacterial infection include the CRP level, PCT level, White cell count, absolute neutrophil counts.

CRP is an acute-phase protein that increases within 4 to 6 hours upon stimulation by pro-inflammatory cytokines and peaks at 36 to 50 hours ${ }^{[13]}$. CRP is often used for early diagnosis of infection and as a bacterial biomarker for sepsis ${ }^{[14]}$, but it has relatively low specificity ${ }^{[13]}$.

PCT is a glycoprotein that consists of 116 amino acids and has a half-life of 20 to 24 hours. Studies using animal models have shown that PCT becomes elevated at 3 to 6 hours and peaks at 6 to 8 hours after bacterial infection ${ }^{[15]}$. A meta-analysis suggested that PCT cannot be used to distinguish between infectious and non-infectious diseases ${ }^{[16]}$.

CURB-65 (confusion, uremia, elevated respiratory rate, hypotension, and age $\geq 65$ ) score, is a clinical prediction rule intended to stratify patients with pneumonia by expected mortality ${ }^{[17,18]}$. But this is a comprehensive measure that does not assess the extent of infection.

CD64 is a receptor for the Fc fragment of immunoglobulin $\mathrm{G}$ and serves as a link between humoral and cellular immunity ${ }^{[19,20]}$. CD64 is mainly distributed on the surface of monocytes, macrophages, and dendritic cells. In neutrophils, CD64 expression is low in the resting state but sharply increases upon activation by a variety of stimuli, including lipopolysaccharide, tumor necrosis factor- $\alpha$, interferon- $\gamma$, and G-CSF ${ }^{[19]}$. When these stimulation factors are absent, it will substantially decrease within 48 hours and will be back to normal baseline values after 7 days ${ }^{[21]}$.

This large retrospective analysis clarifies test characteristics of nCD64, CRP, PCT, WBC, NEUT in diagnosis and prognostic of community-acquired pneumonia. In the retrospective analysis, all community-acquired pneumonia patients were further subdivided into two groups: non severe community-acquired pneumonia(N-SCAP) group and severe community-acquired pneumonia (SCAP) group. The study reports on the evaluation of the usefulness of neutrophil CD64 in elderly patient with community-acquired pneumonia. In our study nCD64, CRP ,PCT, WBC,NEUT level as well as CURB-65 score were higher in severe community-acquired pneumonia patients than in non-severe community-acquired pneumonia. The nCD64 in severe community-acquired pneumonia was significantly upregulated compared with nonsevere community-acquired pneumonia $(P<0.001)$. Evaluation of nCD64 in diagnosing bacterial, AUC $0.787(0.381-0.703)$,cut-off value $>8.95$,Sensitivity 0.743 , Specificity 0.820 PPV 0.645 , NPV 0.879 .The AUC of $\mathrm{nCD} 64$ was higher than that of any other parameter in combination. Similar to previous studies, nCD64 is rapidly upregulated in the early stages of pathogen invasion, it may be particularly valuable in narrowing down the differential diagnosis in emergent situations ${ }^{[22]}$. Increased nCD64 expression initiates and amplifies the immune response to bacterial infection. Therefore plays a vital role in host defense against bacterial infection. Analysis of ROC curves in predicting 28-day mortality in patients, The CURB-65score, nCD64,PCT,CRP levels were higher in non-survivors. nCD64 AUC 0.907(0.843-0.951), cutoff value $>10.43$, Sensitivity 0.1 , Specificity 0.798 PPV 0.463 , NPV 0.100 . There were significant 
differences in nCD64.From the data, we found that nCD64 has been shown to be a better predictor of death, in contrast to CRP, PCT, WBC and NEUT.A recent study demonstrated that $\mathrm{nCD} 64$ has high diagnostic accuracy in recognizing ventilator-associated pneumonia (VAP) ${ }^{[23]}$. Consistent with previous studies, our research demonstrated that nCD64 has a higher AUC compared with PCT, CRP, WBC, NEUT levels. The nCD64 was more sensitive and specific than the CRP ,PCT,WBC,NEUT levels . But there were no significant differences between WBC and NEUT. A recent study demonstrated that the White cell count , absolute neutrophil count can be influenced by many other factors, including trauma, stress, and tumor invasion; therefore, it may not be a suitable biomarker for infection ${ }^{[24,25]}$. We conducted a logistic regression analysis to explore the value of nCD64 expression. Consequence, nCD64 entered the logistic model as independent risk factors of 28-day mortality. The combination of nCD64+ CURB-65 showed the

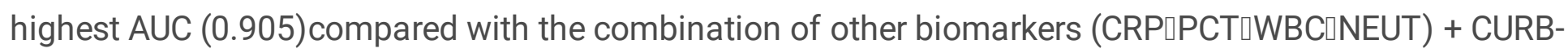
65 score . Because CURB-65 scor does not assess the extent of infection, So nCD64+ CURB-65 score may be a more promising biomarker for identifying infectious etiologies and for predicting mortality.

\section{Limitations}

Our study has several limitations. First, The relatively small sample size and the non-randomized singlecenter design with a short observational period may have resulted in selection bias for clinical data analysis. Second, it is retrospective in nature, so all patients did not have all laboratory values drawn, and this could have introduced bias. Third, Further randomized, multicenter studies with larger sample size and long-term follow-up are needed to validate our results.

\section{Conclusions}

nCD64 expression is a valuable marker for early diagnosis of infection in community-acquired pneumonia, risk stratification and evaluation of prognosis elderly patients with community-acquired pneumonia.

\section{References}

1. Mandell LA, Wunderink RG, Anzueto A, et al. Infectious Diseases Society of America/ American Thoracic Society Consensus Guidelines on the Management of Community-Acquired Pneumonia in Adults. Clin Infect Dis.2007;44: S27-72.

2. KELLY E, MacREDMOND RE, CULLEN G, et al. Community-acquired pneumonia in older patients: Does age influence systemic cytokine levels in community-acquired pneumonia? Respirology. 2009;14 (2):210-216.

3. Yan Liu,Chi Zhang,Chengyu Li,Chunxue Bai,Hongcai Shang.Marked Reduction in 28-day Mortality Among Elderly Patients with Severe Community-acquired Pneumonia: Post Hoc Analysis of a Large Randomized Controlled Trial. Clinical Interventions in Aging.2020;15:2109-2115. 
4. Ramirez JA, Wiemken TL, Peyrani P, Arnold FW, Kelley R, Mattingly WA, et al. Adults Hospitalized With Pneumonia in the United States: Incidence, Epidemiology, and Mortality. Clinical Infectious Diseases. 2017;65:1806-12.

5. Wei Li, Cheng Ding ,Shaojun Yin. Aging increases the expression of lung CINCs and MCP-1 in senile patients with pneumonia. Oncotarget. 2017; 8:108604-108609.

6. Heba E. Hashem, Sherin A. El Masry, Amira M.Mokhtar ,Eman A.Ismail,and Noureldin M. Abdelaal. Valuable Role of Neutrophil CD64 and Highly Sensitive CRP Biomarkers for Diagnostic, Monitoring, and Prognostic Evaluations of Sepsis Patients in Neonatal ICUs. BioMed Research International.2020;2020:6214363.

7. Hoffmann J M Johannes. Neutrophil CD64: a diagnostic marker for infection and sepsis. Clin Chem Lab Med.2009; 47:903-916.

8. J. Burgos1, I. Los-Arcos, D. Álvarez de la Sierra, V. Falcó, A. Aguiló, I. Sánchez, B. Almirante,M. Martinez-Gallo. Determination of neutrophil CD64 expression as a prognostic biomarker in patients with community-acquired pneumonia. European Journal of Clinical Microbiology Infectious Diseases.2016;35:1411-1416.

9. Rupali Patnaik, Afzal Azim, and Vikas Agarwal. Neutrophil CD64 a Diagnostic and Prognostic Marker of Sepsis in Adult Critically III Patients: A Brief Review. Indian Journal of Critical Care.2020;24:12421250.

10. Zhonghua Jie He He Hu Xi Za Zhi.Respiratory Society of Chinese Medical Association.Guidelines for diagnosis and treatment of community-acquired pneumonia in adults in China (2016 edition). Chinese Journal of Tuberculosis and Respiratory Diseases;2016;4:1-27.

11. Cillóniz Catia, Rodríguez-Hurtado Diana, Antoni Torres .Characteristics and Management of Community-Acquired Pneumonia in the Era of Global Aging. Med Sci 2018; 6:35.

12. Chalmers James, Campling James, Ellsbury Gillian, et al. Community-acquired pneumonia in the United Kingdom: a call to action. Pneumonia. 2017; 9:15.

13. Standage SW, Wong HR. Biomarkers for pediatric sepsis and septic shock. Expert Rev Anti Infect Ther. 2011; 9: 71-79.

14. Zhenglong Ye, Hui Zou, Shangxiang Liu,Chengqing Mei, Xiaoliang Chang, Zhiqing Hu,Huanhuan Yang and Yong Wu. Diagnostic performance of neutrophil CD64 index in patients with sepsis in the intensive care unit. Journal of International Medical Research.2019;47:4304-4311.

15. Maruna P, Nedelníková K, Gürlich R. Physiology and genetics of procalcitonin. Physiol Res. 2000; 49: S57-61.

16. Tang BM, Eslick GD, Craig JC, et al. Accuracy of procalcitonin for sepsis diagnosis in critically ill patients: systematic review and meta-analysis. Lancet Infect Dis 2007; 7: 210-217.

17. Annette Ilg, Ari Moskowitz, Varun Konanki, Parth V. Patel, RN, Maureen Chase, Anne V. Grossestreuer, Michael W. Donnino. Performance of the CURB-65 Score in Predicting Critical Care Interventions in Patients Admitted With Community-Acquired Pneumonia. Ann Emerg Med. 2019 ;74:60-68. 
18. Mandell LA, Wunderink RG, Anzueto A, Bartlett JG, Campbell GD, Dean NC, Dowell SF, File TMJr, Musher DM, Niederman MS et al. Infectious Diseases Society of America/American Thoracic Society consensus guidelines on the management of community-acquired pneumonia in adults. Clin Infect Dis. 2007; 44: S27-72.

19. Chen Q, Shi J, Fei A, et al. Neutrophil CD64 expression is a predictor of mortality for patients in the intensive care unit. Int J Clin Exp Pathol. 2014; 7: 7806-7813.

20. Elawady S, Botros SK, Sorour AE, et al. Neutrophil CD64 as a diagnostic marker of sepsis in neonates. J Investig Med. 2014; 62: 644-649.

21. Schiff DE, Rae J, Martin TR. Increased phagocyte Fc gamma RI expression and improved Fc Gammareceptor mediated phagocytosis after in vivo recombinant human interferon-gamma treatment of normal human subjects. Blood. 1997; 90:3187-3194.

22. Rupali Patnaik, Afzal Azim, and Vikas Agarwal. Neutrophil CD64 a Diagnostic and Prognostic Marker of Sepsis in Adult Critically III Patients: A Brief Review. Indian Journal of Critical Care.2020;24(12):1242-1250.

23. Wen-peng Yin, Jia-bao Li, Xiao-fang Zheng, Le An, Huan Shao, Chun-sheng Li. Effect of neutrophil CD64 for diagnosing sepsis in emergency department. World J Emerg Med, 2020;11: 79-86.

24. Zaeksh M, Sedaghat F, Heidarzadeh A, et,al. Diagnostic value of IL-6,CRP,WBC,and absolute neutrophil count to predict serious bacterial infection in febrile infants. Acta Med Iran 2015;53:408411.

25. Zhenglong Ye, Hui Zou, Shangxiang Liu,Chengqing Mei, Xiaoliang Chang, Zhiqing Hu,Huanhuan Yang and Yong Wu. Diagnostic performance of neutrophil CD64 index in patients with sepsis in the intensive care unit. Journal of International Medical Research.2019;47(9):4304-4311.

\section{Figures}



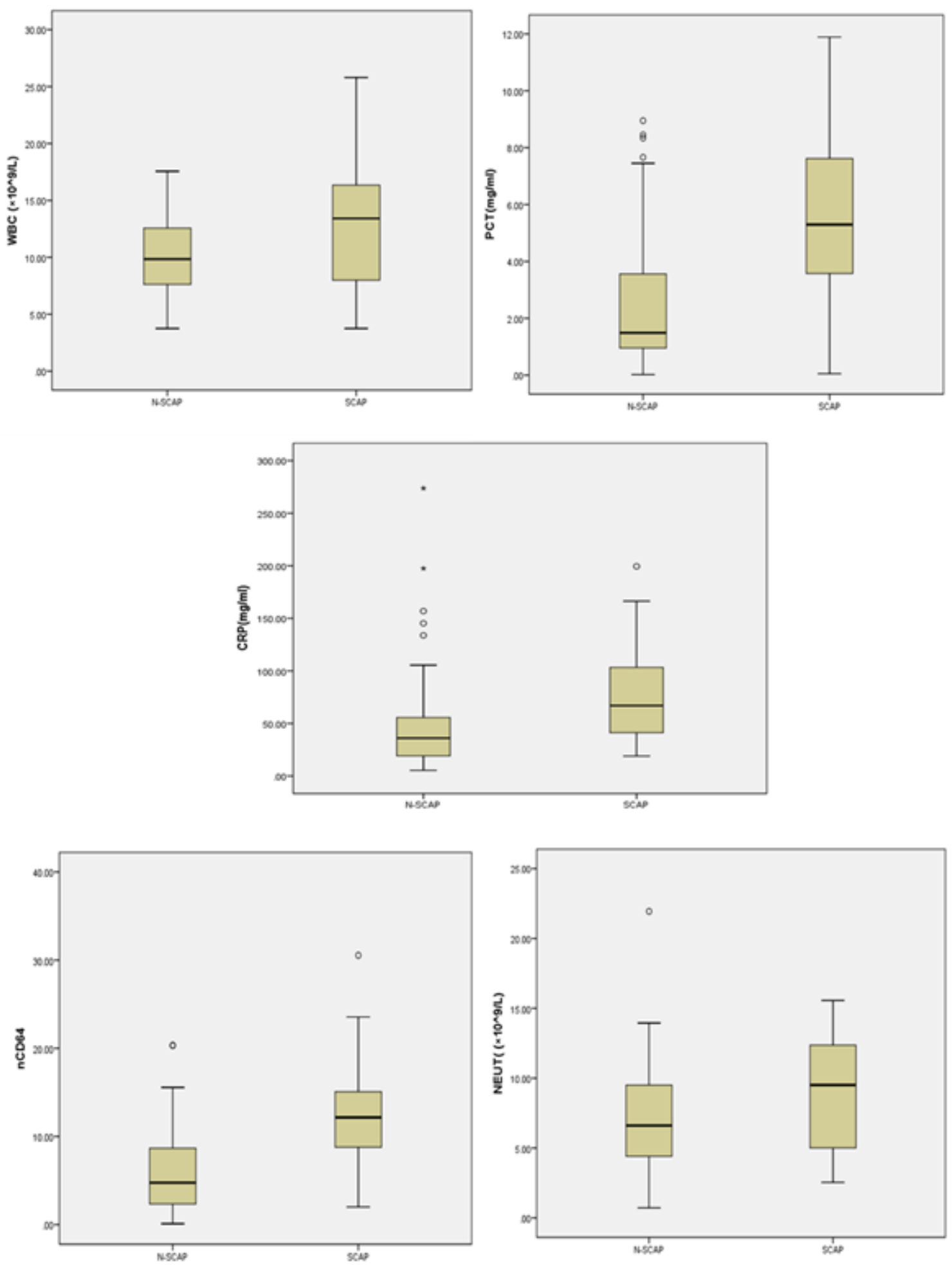

Figure 1

The nCD64, CRP,PCT,WBC,NEUT levels in two group. The nCD64, CRP ,PCT, WBC, NEUT of the patient groups were significantly higher compared with the N-SCAP $(P<0.05)$. The SCAP group had the highest level of these parameters. 


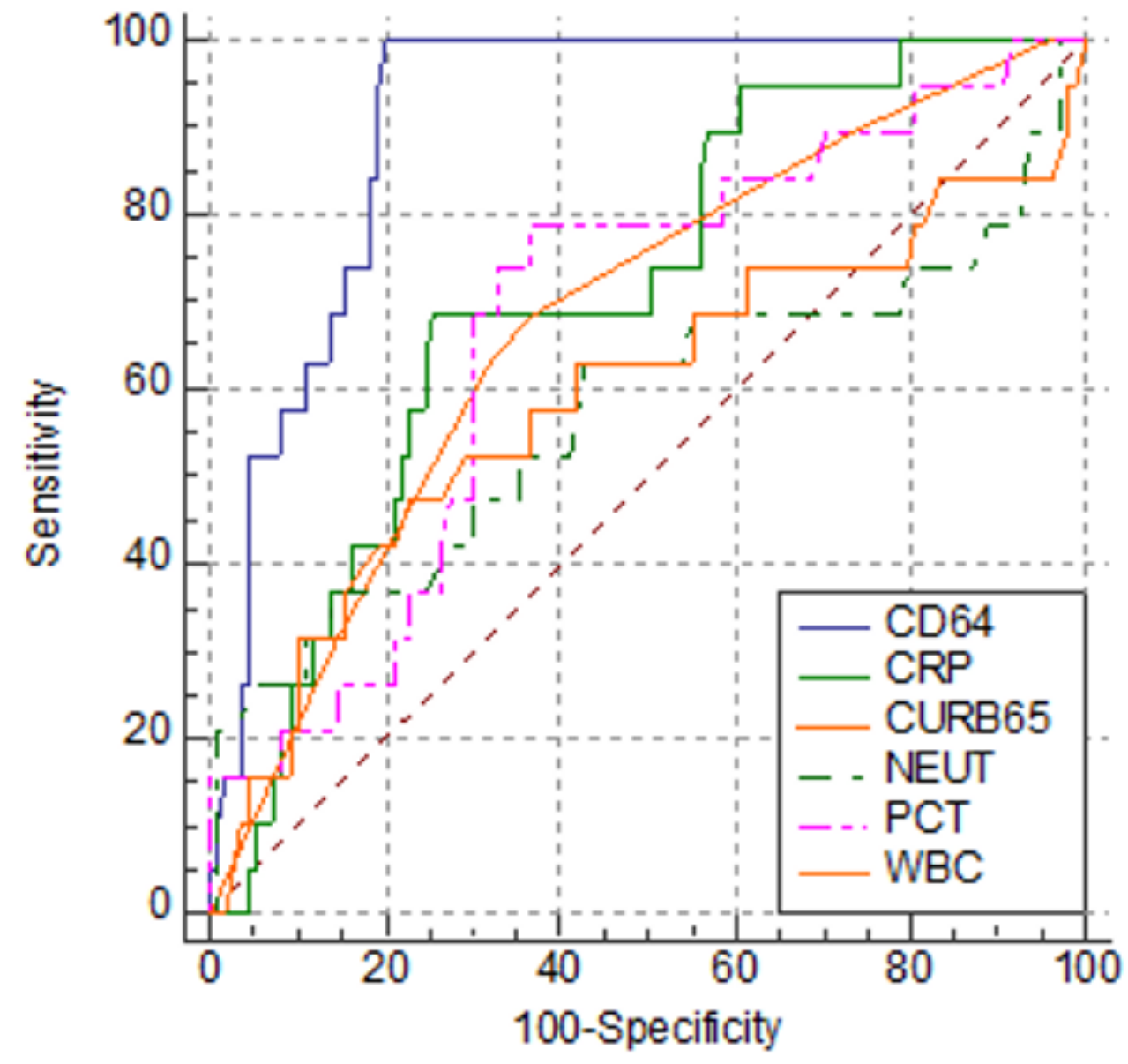

Figure 2

The ROC curves of nCD64, PCT, CRP, WBC and NEUT for prognosis. The AUC of nCD64 was the highest (0.907), followed by CRP (0.710) PCT (0.678), WBC (0.592) and NEUT (0.573). There were significant differences between nCD64 and CRP or PCT $(\mathrm{P}<0.001)$. 


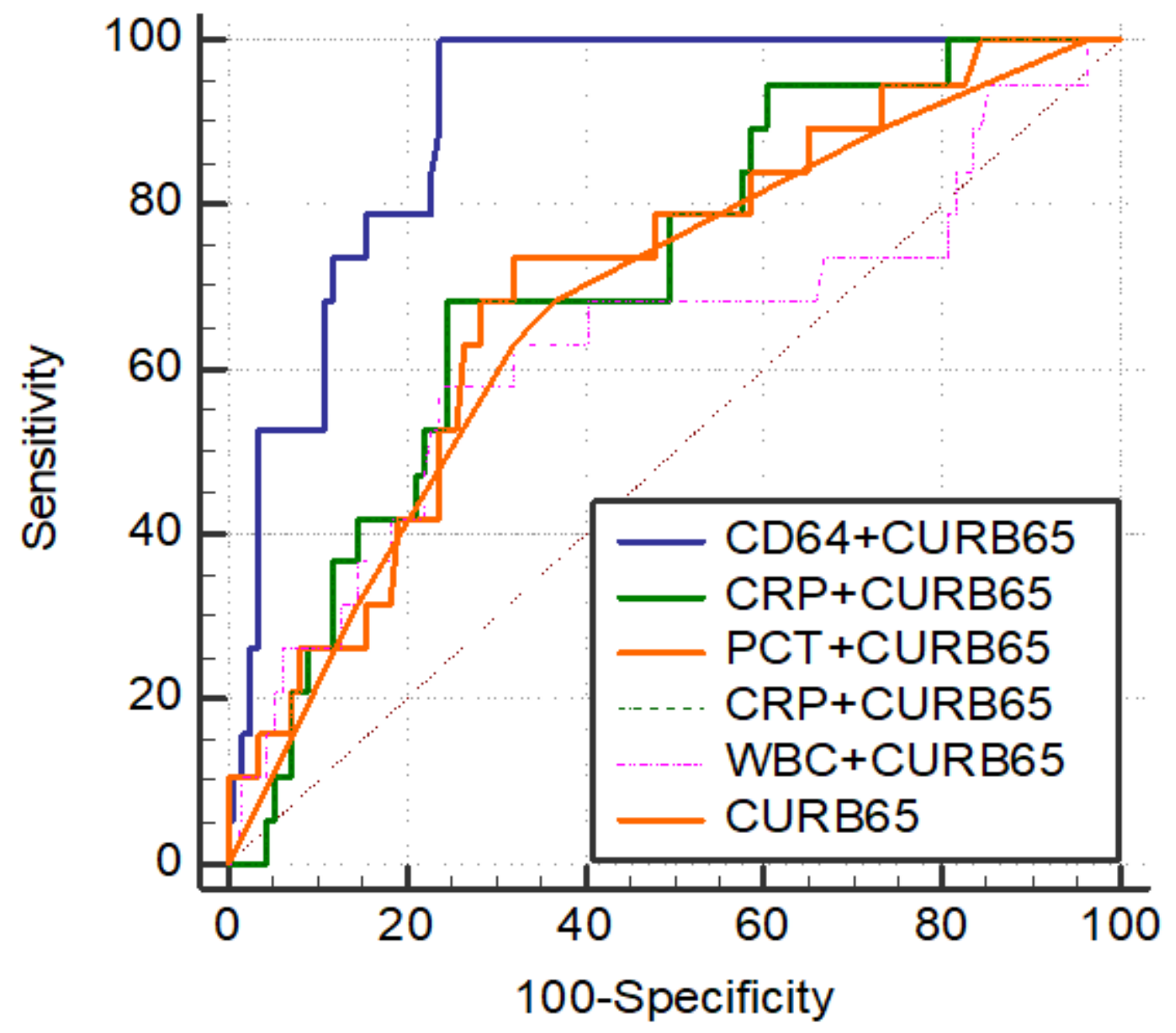

Figure 3

The ROC curves of nCD64+CURB-65 score, PCT+CURB-65 score, CRP+CURB-65 score, WBC+CURB-65 score , NEUT+CURB-65 score and CURB-65 score for prognosis. The AUC of nCD64+CURB-65 score was the highest (0.905),followed by CRP +CURB-65 score (0.712),PCT +CURB-65 score (0.704), WBC+CURB-65 score (0.632) , NEUT+CURB-65 score (0.636). CURB-65 score (0.680). There were significant differences between nCD64+CURB-65 score ,CRP +CURB-65 score ,PCT+CURB-65 score and CURB-65 score $(P<0.001)$. 